UDC 004.75

\author{
*Sarsembayev M., Turdalyuly M., Omarova P. \\ Faculty of Mechanic-Mathematical, \\ al-Farabi Kazakh National University, Almaty, Kazakhstan \\ "e-mail: magatrone@mail.ru
}

\title{
Cloud data-processing system for the automated generation of combustion models
}

\begin{abstract}
Demands of energy for the twenty-first century and beyond will require the development of alternative, renewable fuel sources. Validating an alternative fuel source or fuel additive inherently comes from a posteriori knowledge: run a series of experiments whose results will assist in determining whether additional experiments should be conducted. Unfortunately, this knowledge is acquired at the expense of the fuel.The development of data-processing system of this type will not only timely and promptly provide engineers predictive chemical models of combustion processes of real fuels, but also will create a basis for organizing and coordination of fundamental data on the thermodynamic and kinetic properties of the hydrocarbon molecules, mechanisms of combustion of the individual components of real fuels and mixtures thereof. As an example, it suffices to mention that due to the inconsistency of data used by different research groups at the same time there are multiple versions of this simple combustion of fuel such as hydrogen, not to mention the much more complex hydrocarbon fuels.PrIMe (Process Informatics Model)- is a new approach for developing predictive models of chemical reaction systems that is based on the scientific collaborator paradigm and takes full advantage of existing and developing cyber infrastructure.
\end{abstract}

Key words: Cloud data-processing system, combustion models,PriMe, Modeling, combustion, global systems, web-based application, collaborative science.

Develop and maintain up to date detailed predictive models of chemical combustion is impossible without real software tools for analysis, processing and storage of large volumes of initial theoretical and experimental data. Furthermore, the process of continuous renewal of existing and new generation of chemical models is associated with a large number of computations.

Analysis of recent publications on the subject of the development of the kinetic schemes actual fuel combustion suggests that the current system, fully implement the set of computational modules and databases to automate this work, are not used because of the absence. Basically, researchers use computational modules that could be the private components of such a system.

Private task generating kinetic schemes combustion of hydrocarbons was solved a number of research groups $[3,6]$. RMG is the most advanced system among the cited systems [7] (reaction mechanism generation), the main advantage of which is extensible architecture. The essence of the extensible architecture is to create a hierarchy of derived classes of reaction centers in the molecules of hydrocarbons (and the corresponding rate constants of elementary processes) and the corresponding database, allowing the user to expand the database that is automatically reflected on the results of the hydrocarbon generation kinetic combustion schemes. Unfortunately, the main disadvantage of this system is the lack of direct assessment of the uncertainties in the rate constants of reactions of classes that give her isolated from the follow-up mechanism for the advancement of the generated.

Of particular importance of the presence of chemical mechanisms it has predictive in modeling real-world fuel combustion processes. This is due not only to the high importance of practical problems to be solved, but above all to the fact that computer modeling using detailed chemical information, is an essential part of the modern cycle development and optimization of power plants, which is essential to the achievement of the required parameters for today.

The task of developing and maintaining up to date detailed predictive self-consistent models of real chemical fuel combustion requires today address the following issues: effective use of the latest models of experimental and theoretical data on

Printed in Kazakhstan 
the thermodynamic and chemical properties of hydrocarbon molecules; coherence patterns (property of the same materials and basic processes in different models must be the same); use to verify the model of the widest possible range of experimentally measured characteristics of the selected burning fuel (or fuel group); chemical recovery of values of model parameters on the basis of direct and indirect experimental measurements, the use of information about the accuracy of the model parameters for the analysis of uncertainty propagation and evaluation of the reliability of results in multiscale modeling of engineering.

Solution of these problems requires the analysis, processing and storage of large volumes of initial theoretical and experimental data. Furthermore, the process of continuous renewal of existing and new generation of chemical models is associated with a large number of computations. These problems can not be solved without the involvement of a modern information infrastructure: optimal computational algorithms and data management technologies and computing tasks in heterogeneous distributed systems. The scientific problem to be solved by the project aims is to develop the theoretical foundations of a new generation of data-processing system for the automated design of chemical models of real burning hydrocarbon fuels with the assessment of the reliability of the simulation results.

The closest prototype of the universal system, implemented to date, is a web service PrIMe [1,2], created and supported by the group of Professor M. Frenklach. This system implements the functionality associated optimization of kinetic schemes and disseminating the analysis of uncertainties in the modeling process. It is worth noting that at the heart of this system lies Data Collaboration concept, the essence of which is to collect all available direct and indirect experimental information on the combustion process and its subsequent use for the prediction of the required properties of combustion processes. This concept is universal and can be extended to other subject areas. The main limitation of this system is the lack of libraries of models of combustion processes and efficient parallel solvers running on high-performance systems. Also, the possibility of a system limited to a single set of methods for inverse kinetic problem (methods of sequential quadratic programming) solutions, whose capacity may not be enough in the analysis of combustion kinetic schemes of large hydrocarbons.
PrIMe - Process Informatics Model- is a new approach for developing predictive models of chemical reaction systems that is based on the scientific collaboratory paradigm and takes full advantage of existing and developing cyber infrastructure. The primary goals of PrIMe are collecting and storing data, validating the data and quantifying uncertainties, and assembling the data into predictive models with quantified uncertainties to meet specific user requirements. The principal components of PrIMe include: a data Depository, which is a repository of data provided by the community $[4,8]$, a data Library for storage of evaluated data, and a set of computer-based tools to process data and to assemble data into predictive models. Two guiding principles of PrIMe are: open membership- a qualified individual or industrial organization can register to participate in the project; and open source- all submitted data, tools and models will be in the public domain.

The objective of this project was to sustain and extend the development of the PrIMe cyber infrastructure for the practical use by the Combustion community. PrIMe (Process Informatics Model) is a new approach for developing predictive models of chemical reaction systems that is based on the scientific col laboratory paradigm. The primary goals of PrIMe are collecting and storing data, validating the data and quantifying uncertainties, and assembling the data into predictive models with quantified uncertainties to meet specific user requirements. The principal elements of PrIMe include: a data Warehouse which is a repository of data provided by the community, a data Library which archives community-evaluated data, and computer-based tools to process data and to assemble data into predictive models.

Optimizing combustion efficiency and understanding the mechanisms that prevent full energy utilization of fuels relies on detailed knowledge of the underlying physics and chemistry [7]. These systems are generally complex enough that models have been used to explore the effectof different feed and reactor conditions and have been successful in optimizing fuel mixtures and combustor performance. However, the models are extremely complex and often controversial. The data, which parameterize the models and are compared to model predictions, are themselves complex and often open to interpretation. Further, they are developed by multiple labs using different technologies. To keep track of models, parameters, 
and data in an integrated framework has proven a necessity in the field of Combustion. The PrIMe initiative is designed to fill this need. In its scientific content, PrIMe is a system approach aimed at establishing the infrastructure, both scientific and $\mathrm{CI}$, in support of developing predictive models of combustion.

The initial phase and development of PrIMe CI has focused on underlying chemical reaction models. There are several important reasons for this strategy. First, modeling of a combustion process begins with a reaction model, which determines the concentrations of chemical specifies and the heat flux, and hence it is only natural to start the new development from this founding stage. It has been our experience1 that most disagreements between models and experiments and most controversies begin with and trace to the selected reaction model. Another factor for starting with reaction models is the fact that chemical kinetics has accumulated much needed data and the missing data can be evaluated using quantum and reaction-rate theories. And finally, the scientific underpinning of the process, also illustrating the feasibility of the approach, has been piloted by the GRI-Mech project.

The PrIMe Workflow Application (PWA) is a web-based application that unifies the components of PrIMe into a single interface [5]. The purpose of this document is to describe the PrIMe Workflow Application architecture and its internal structure. The functionality of PWAcomponents is depicted in the form of Use Case diagrams. Class diagrams, consistencydiagrams, data-base scheme, and components diagrams are used to demonstrate systemdesign and component interaction.

The common structure of PrIMe is consists of the following components:

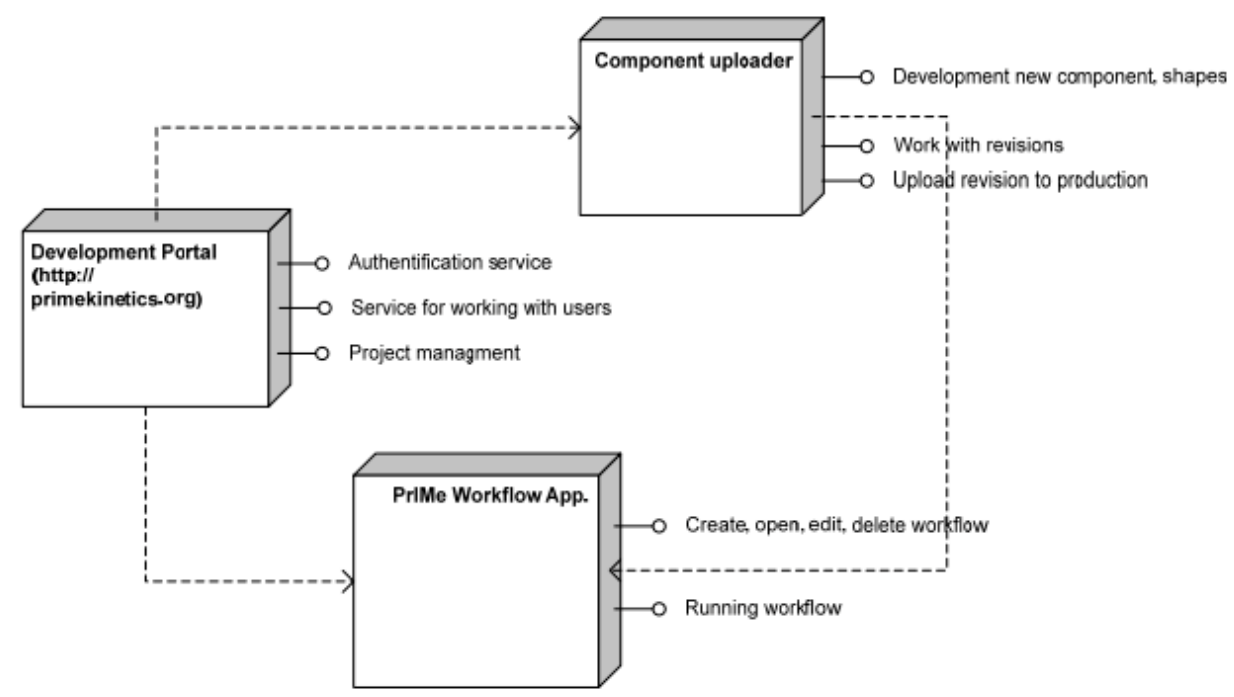

Figure - Components of the PrIMe structure

1. PrIMe Portal is responsible for system user management. It implements user authorization and authentication services, assigns user roles, and manages user permissions. Additionally, it enables users to collaborate. In the PrIMe portal one can find information such as the latest changes, documentation, and operating instructions. The Development Portal provides an interface for two additional systems - Scientific Component Uploader and PrIMe Workflow Application.

2. Scientific Component Uploader (SCU) is used to develop and deploy new scientific components. It allows the scientific component developer to upload a new scientific component, assign resources to components, and edit properties and configuration information of his/her previously developed components. All changes made by developers are stored as separate revisions, allowing a developer to open and edit any existing revisions. Only a user with administrator privileges can create a new revision and deploy it to the PrIMe Workflow Application (PWA).

3. PrIMe Workflow Application (PWA) is the "environment" where a user creates and executes 
scientific workflow projects. The scientific workflow project is built using preconfigured scientific components that are linked together in a network. The user can set input and output information for each scientific component and, if applicable, the user.

\section{References}

1. M. Frenklach, Transforming data into knowledge-Process Informatics for combustion chemistry, Proceedings of the Combustion Institute, Vol 31, Issue 1, January 2007, Pages 125-140

2. Susnow, R.G.; Dean, M.A.; Green, W.H.; Peczak, P.; Broadbelt, L.J. Rate-Based Construction ofKinetic Models for Complex Systems. J. Phys. Chem. A 1997, 101, 3731-3740

3. Warth, V.; Battin-Leclerc, F.; Fournet, R.; Glaude, P.A.; Come G.M.; Scacchi, G. Computer based generation of reaction mechanisms for gasphase oxidation. Comput. Chem. 2000, 24, 541-560

4. Rakiewicz A. and Truong. T. N. Application of chemical graph theory for automated mechanism generation. J. Chem. Inf. Comput. Sci., 2003, 43 (1), pp 36-44

5. E. Ranzi, T. Faravelli, P. Gaffuri, and A. Sogaro. Low-temperature combustion: automatic generation of primary oxydation reaction and lumping procedures. Combustion and Flame, 102:179, 1995

6. William H. Green, Joshua W. Allen, Beat A. Buesser, Robert W. Ashcraft, Gregory J. Beran, Caleb A. Class, Connie Gao, C. Franklin Goldsmith, Michael R. Harper, AmritJalan, Murat Keceli, Gregory R. Magoon, David M. Matheu, Shamel S. Merchant, Jeffrey D. Mo, Sarah Petway, Sumathy Raman, Sandeep Sharma, Jing Song, YurySuleymanov, Kevin M. Van Geem, John Wen, Richard H. West, Andrew Wong, Hsi-Wu Wong, Paul E. Yelvington, Nathan Yee, Joanna Yu; "RMG - Reaction Mechanism Generator v4.0.1", 2013

7. Benson, S.W. Thermochemical Kinetics: Methods for the Estimation of Thermochemical Data andRate Parameters2d ed., John Wiley \& Sons, New York, 1973

8. www.primekinetics.org 